\title{
Introduction to the AfricaGEO 2018 Conference Special Issue of the South African Journal of Geomatics
}

\author{
Peter MU Schmitz ${ }^{1,2}$, Antony K Cooper ${ }^{3,4}$, Sanet Carow ${ }^{1}$, Peter Newmarch ${ }^{5}$, Julian Smit ${ }^{6}$ \\ ${ }^{1}$ Department of Geography, University of South Africa, Florida, South Africa. \\ schimpmu@unisa.ac.za \\ ${ }^{2}$ Fakultät für Vermessung, Informatik und Mathematik, Hochschule für Technik Stuttgart, \\ Stuttgart, Germany \\ ${ }^{3}$ Built Environment, CSIR, Pretoria, South Africa, acooper@csir.co.za \\ ${ }^{4}$ Centre for Geoinformation Science, University of Pretoria, Pretoria, South Africa \\ ${ }^{5}$ South African Geomatics Council, South Africa \\ ${ }^{6}$ Geomatics Division, University of Cape Town, Cape Town, South Africa
}

The AfricaGEO 2018 Conference, with the theme Developing Geomatics in Africa: Data fusion - Driving Value and Policy, is being held at the Caesars Palace Convention Centre, City of Ekurhuleni, South Africa, from 17 to 19 September 2018. The conference consists of a peerreviewed academic paper track, an industry paper track, plenary presentations, workshops, tutorials, lightning talks, demonstrations and an exhibition. The selection for the Industry Track was made on abstracts submitted and some authors also submitted full papers. These abstracts and papers have been published online at: http://www.africageoproceedings.org.za/

The ring-fenced, peer-reviewed Academic Track of AfricaGEO 2018 is independent of the Industry Track. Submissions for the Academic Track underwent a double-blind peer review based on full papers. Papers were reviewed by at least two independent reviewers each, on the basis of significance, contribution, relevance, correctness, originality, clarity and structure. Initially, 26 papers were submitted and of these, 4 were rejected as not being suitable for the Academic Track (some of these were passed on to the Chair of the Industry Track for their consideration). The results and comments from the referees were sent back to the authors, for them to revise their papers appropriately and to resubmit their papers. After evaluating the resubmitted papers, the editors rejected a further three papers because the authors did not submit papers that had been revised suitably.

This review process resulted in 21 papers considered suitable for inclusion in the Proceedings of the peer-reviewed Academic Track of AfricaGEO 2018. The ISBN is 978-0-620-80873-6 for these proceedings, published separately online at: http://www.africageoproceedings.org.za/ 
The Editor of the South African Journal of Geomatics agreed to a special issue of the journal for selected papers from the peer-reviewed Academic Track of AfricaGEO 2018, should there be any of sufficient quality for inclusion in the journal. After reviewing again, the papers selected for the proceedings of the Academic Track, eight papers were identified by the editors of this special issue as being of a sufficient quality. The authors were asked to make further improvements to their papers and subsequently, one paper was declined. The following is a brief summary of the seven papers included in this special issue of the South African Journal of Geomatics.

Hull and Whittal, in "Filling the Gap: Customary Land Tenure Reform in Mozambique and South Africa", used a conceptual framework with 87 indicators they had previously developed for guiding cadastral systems development in customary land rights contexts, to assess land tenure reform in Mozambique. They used their analysis to make ten recommendations that could apply to South Africa.

There are three papers that use remote sensing extensively. Ritchie, Debba, Lück-Vogel and Goodall, in "Assessment of Accuracy: Systematic Reduction of Training Points for Maximum Likelihood Classification and Mixture Discriminant Analysis (Gaussian and t-distribution)", compasred the accuracies of Maximum Likelihood Classification (MLC) and its extension, Mixture Discriminant Analysis (MDA). They found that MDA produced better results when sufficient training points are available.

Then, Fundisi and Tesfamichael, in "Assessing the effect of band selection on accuracy of pansharpened imagery application to young woody vegetation mapping", investigated how well pansharpening of different spectral bands of Landsat 8 imagery affected discriminating young woody vegetation from other land cover. Pansharpening produced small improvements.

Phiri, Shiferaw and Tesfamichael, in "Modelling the relationship between groundwater depth and NDVI using time series regression with Distributed Lag Models", used data from two groundwater monitoring stations to compare groundwater depth with Normalized Difference Vegetation Index (NDVI) data. They found that recent (first lag) NDVI is a significant predictor of NDVI for both grassland and shrubland, while first lag groundwater depth is a significant predictor for grassland, but second lag depth is for shrubland.

There are two papers focusing on modelling, both coincidentally in the Western Cape. Tizora, Le Roux Mans and Cooper, in "Adapting the DynaCLUE model for simulating land use and land cover change in the Western Cape Province", assessed the accuracy of simulating land use and land cover changes at a provincial scale, using the Dyna-CLUE with spatial policies and 
restrictions; land-use type conversions; land use requirements (demands) and location characteristics as parameters. Using base data from 1990, they generated 23 simulation maps through to 2014, with the final simulation agreeing well with actual 2013/4 data, both visually and statistically.

Wallace, in "Spatializing the modelled impacts of future climate change - a case study on wheat in the Western Cape", modelled crop yields for 21 zones in the province (which is highly diverse geologically, topographically and climatically), based on the downscaled results of global climate models. Using a geographical information system greatly facilitated the integration and analysis of the multi-scale data and displaying the results on maps.

Madubedube, Rautenbach and Coetzee, in "Investigating the impact of different types of directions on wayfinding efficiency in an informal settlement", assessed how well participants could navigate through an irregularly structured informal settlement (without street names), using turn descriptors and landmarks, or using cardinal directions and distances. The participants tended to prefer turn descriptors (left-right directions) and performed better with them.

Finally, we would like to thank all the authors who submitted papers to AfricaGEO 2018, because we need quality submissions to ensure the conference is a success. We are pleased by the overall quality of the papers submitted and we hope that you will find the conference rewarding. We would also like to acknowledge and thank the anonymous reviewers for their hard work in refereeing papers for the Academic Track of AfricaGEO 2018 and for this special issue of the South African Journal of Geomatics, 